\title{
Contribution à la caractérisation physico-chimique des déchets de deux marchés de la commune de Port-au-Prince (Haïti) : propositions de traitements
}

\author{
Fred Alix COUTIN', Féniel PHILIPPE', Paul VERMANDE² \\ I. Département de génie rural, Faculté d'agronomie et de médecine vétérinaire, \\ Université d'État d'Haïti, BP | 44 I, Damien, Port-au-Prince, Haïti \\ 2. Laboratoire d'analyse environnementale des procédés et systèmes industriels (LAEPSI), INSA, Lyon
}

Pour tout contact : paul.vermande@insa-lyon.fr

\section{Résumé}

La gestion des déchets implique une connaissance précise de leur composition ainsi que leur quantification. Le Service métropolitain de collecte des résidus solides (SMCRS) dispose déjà de données sur le tonnage des déchets de marchés, mais notre étude vise à fournir les informations nécessaires sur leur composition et leur niveau de toxicité éventuelle. Des solutions pour une meilleure gestion pourront alors être proposées. Les marchés « Croix-des-Bosssales » et « Salomon » de la commune de Port-au-Prince qui sont parmi les plus importants (environ 100 tonnes/jours) ont été retenus comme sites pour cette étude.

En plus de la détermination de la composition des déchets, les analyses suivantes ont été menées conformément aux normes ISO 17025 : I'humidité, le pH et la conductivité électrique, la matière organique, les ions minéraux et quelques métaux lourds.

Les résultats obtenus permettent de conclure qu'un traitement approprié pour ces déchets de marché pourrait être le compostage : la forte teneur en matière organique et la faible quantité de métaux lourds sont en effet des arguments intéressants.

\section{Mots clés :}

déchets de marché, caractérisation physique et chimique, compostage

\section{Introduction}

La gestion des déchets solides dans les villes des pays en développement (PED) constitue l'un des défis majeurs de la présente décennie (Zahrani et al., 2006). Les problèmes d'évacuation, de traitement et de gestion des déchets municipaux dans les PED demeurent cruciaux (Charnay, 2005). Mal gérés, ces déchets dont la quantité n'arrête pas d'augmenter peuvent présenter des risques pour l'environnement et la santé. Les responsables politiques optent donc pour des stratégies qui répondent non seulement aux conditions socioéconomiques, culturelles et technologiques des PED, mais aussi aux caractéristiques du gisement de ces pays.

En Haïti, le Service métropolitain de collecte des résidus solides (SMCRS) dispose déjà de données, quoique partielles, sur le tonnage des déchets urbains. La caractérisation physico-chimique des déchets de marchés qui est présentée fournit des données sur leur composition et leur niveau de toxicité en vue d'aider les responsables à avoir une démarche rationnelle dans la collecte et les traitements de ces déchets.

\section{Matériel et méthodes}

\section{Protocole d'échantillonnage}

La campagne de prélèvement a été réalisée en octobre 2004 mais elle peut être considérée comme représentative, car les déchets des marchés n'évoluent pas beaucoup au cours de l'année, malgré une pluviosité non uniforme. Cette campagne concerne deux marchés de la commune de Port-au-Prince : « Croix-des-Bossales », le plus grand marché de la capitale (35 $000 \mathrm{~m}^{2}$ avec 150 à $200 \mathrm{~m}^{3} / \mathrm{j}$ de déchets, et le marché «Salomon », avec 40 à $60 \mathrm{~m}^{3} / \mathrm{j}$ (source: SMCRS, exercice 2004/2005, non publié). Ces marchés se situent respectivement aux entrées nord et sud de la commune de Port-au-Prince. Ils reçoivent les produits provenant de tout le pays. Trois échantillons de 10 à $15 \mathrm{~kg}$ de déchets par marché ont été prélevés après brassage des tas, homogénéisation et quartage ; les échantillons sont identifiés CI, C2, C3 , SI, S2, S3. Chaque objet constitutif des déchets est prélevé puis fragmenté pour passer au tamis de $2 \mathrm{~mm}$ de diamètre. Les objets sont pesés séparément puis mélangés entre eux pour donner les échantillons de laboratoire sur lesquels les différents tests et analyses ont été réalisés.

\section{Caractérisation physique des déchets de marché}

La composition physique des déchets de marchés a été déterminée grâce à un tri soigné des divers composants constitutifs ; on a déterminé le pourcentage de chacun par rapport au poids brut humide. La caractérisation physique de 
ces déchets s'est limitée à déterminer quelques paramètres comme l'humidité, le $\mathrm{pH}$ et la conductivité des solutions obtenues après macération d'une quantité de déchets (20 g) dans l'eau ( 100 millilitres) pendant 24 heures.

Le pourcentage d'humidité a été obtenu par chauffage à l'étuve $\left(105^{\circ} \mathrm{C}\right)$ pendant 24 heures : $45 \mathrm{~g}$ d'un échantillon étaient répartis en trois fractions d'environ $15 \mathrm{~g}$ chacune.

\section{Caractérisation chimique des déchets de marché}

Une calcination a été effectuée au four à moufle sur $3 \mathrm{~g}$ de matières sèches par échantillon pendant 4 heures à $600^{\circ} \mathrm{C}$. Ceci permet d'obtenir la teneur en matière organique.

On a déterminé les chlorures après une extraction à l'eau de 12 g dans $500 \mathrm{ml}$ d'eau distillée. Les mesures ont été effectuées à l'aide d'un spectrophotomètre Nova 600.

La minéralisation de $0,5 \mathrm{~g}$ de dechets séchés et broyés dans $5 \mathrm{ml}$ du mélange d'acides chlorhydrique et nitrique concentrés a permis la mise en solution des métaux alcalins et alcalinoterreux ainsi que celle des métaux lourds. La spectrométrie d'absorption atomique a alors été utilisée pour le dosage des éléments « majeurs » $(\mathrm{Na}, \mathrm{K}, \mathrm{Ca}, \mathrm{Mg})$ et de ceux qui sont à l'état de «traces » $(\mathrm{Pb}, \mathrm{Co}, \mathrm{Ni}, \mathrm{Cr}, \mathrm{Zn}$, etc.).

L'ensemble des tests a été réalisé conformément à la norme ISO 17025.

\section{Résultats et discussions}

\section{Caractéristiques physiques des déchets de marché}

Dans tous les échantillons, la présence de résidus végétaux et animaux est très significative car, dans la majorité des cas, elle dépasse les 70 \% (tableau I). Nous avons également observé, lors de notre visite de prospection, des emballages métalliques liés à la commercialisation du lait et du concentré de tomates ainsi que des morceaux de vêtements. Ces catégories n'ont pas été répertoriées dans notre collecte. Ceci est dû sans doute au fait que ce sont des marchés d'approvisionnement des commerçants et non de consommateurs. Au sein des déchets ménagers de la ville, la présence de ces types de déchets est bien évidemment observée (tableau 3).

\begin{tabular}{|l|l|l|l|l|l|}
\hline Echantillons & $\begin{array}{l}\text { Restes } \\
\text { végétaux } \\
\text { et } \\
\text { animaux }\end{array}$ & $\begin{array}{l}\text { Matières } \\
\text { plastiques } \\
\text { de } \\
\text { couleurs } \\
\text { variées }\end{array}$ & $\begin{array}{l}\text { Cartons et } \\
\text { papiers } \\
\text { d'emballages, } \\
\text { papier journal }\end{array}$ & $\begin{array}{l}\text { Charbon } \\
\text { de bois }\end{array}$ & $\begin{array}{l}\text { Bouteilles } \\
\text { ou verres }\end{array}$ \\
\hline $\mathrm{S}_{1}$ & 95,87 & 3,25 & 0,88 & 0,00 & 0,00 \\
\hline $\mathrm{S}_{2}$ & 84,43 & 7,39 & 8,18 & 0,00 & 0,00 \\
\hline $\mathrm{S}_{3}$ & 94,78 & 5,22 & 0,00 & 0,00 & 0,00 \\
\hline $\mathrm{C}_{1}$ & 71,92 & 0,00 & 2,86 & 25,22 & 0,00 \\
\hline $\mathrm{C}_{2}$ & 87,33 & 2,50 & 10,17 & 0,00 & 0,00 \\
\hline $\mathrm{C}_{3}$ & 41,10 & 2,12 & 5,72 & 33,45 & 17,61 \\
\hline Moyenne & 79,22 & 3,41 & 4,63 & 9,79 & 2,93 \\
\hline Ecart-type & 11,53 & 2,57 & 3,52 & 12,62 & 6,56 \\
\hline
\end{tabular}

Tableau I : Résultats de l'analyse physique $100 \%$ déchets

L'humidité des déchets de marché est de $75 \%$, valeur moyenne sur les 6 échantillons (tableau 2). Ce pourcentage est un élément intéressant pour la fabrication de compost de qualité car $50 \%$ d'humidité sont nécessaires dans les déchets lors de la mise en fermentation aérobie. Cette humidité contribue au développement de micro-organismes hydrophiles, à la décomposition de la matière organique, mais elle peut aussi contribuer, au cours du stockage des déchets, à la production de lixiviats qui s'infiltreront dans le sous-sol (transfert possible de polluants).

\begin{tabular}{|c|c|c|}
\hline Eléments & Valeurs & Unités \\
\hline $\mathrm{Ca}$ & $0,5-2,9$ & $\%$ du total des déchets \\
\hline $\mathrm{Mg}$ & $<1$ & idem \\
\hline $\mathrm{Na}$ & $<1$ & idem \\
\hline $\mathrm{K}$ & $<1$ & idem \\
\hline $\mathrm{Co}$ & $1-6$ & $\mathrm{mg} / \mathrm{kg}$ de MS \\
\hline $\mathrm{Cu}$ & $<20$ & $\mathrm{mg} / \mathrm{kg}$ de $\mathrm{MS}$ \\
\hline $\mathrm{Cr}$ & $34-187$ & $\mathrm{mg} / \mathrm{kg}$ de $\mathrm{MS}$ \\
\hline $\mathrm{Mn}$ & $38-138$ & $\mathrm{mg} / \mathrm{kg}$ de $\mathrm{MS}$ \\
\hline $\mathrm{Pb}$ & $4-151$ & $\mathrm{mg} / \mathrm{kg}$ de MS \\
\hline $\mathrm{Zn}$ & $8-152$ & $\mathrm{mg} / \mathrm{kg}$ de MS \\
\hline Matières organiques & $40,8-90,9$ & $\%$ du total \\
\hline PH & $5,0-6,3$ & - \\
\hline Conductivité & $1,96-3,31$ & $\mu \mathrm{s} / \mathrm{cm}$ \\
\hline Humidité & 75,5 & $\%$ d'eau \\
\hline Nitrates & $<500$ & $\mathrm{mg} / \mathrm{kg}$ de $\mathrm{MB}$ \\
\hline Sulfates & $900-2300$ & $\mathrm{mg} / \mathrm{kg}$ de $\mathrm{MB}$ \\
\hline Chlorures & $1000-2500$ & $\mathrm{mg} / \mathrm{kg}$ de $\mathrm{MB}$ \\
\hline Ammonium & $0,8-6$ & $\mathrm{mg} / \mathrm{kg}$ de $\mathrm{MB}$ \\
\hline $\begin{array}{l}\text { Résidus animaux et } \\
\text { végétaux }\end{array}$ & 79,2 & $\%$ de la MB \\
\hline Matières plastiques & 3,4 & $\%$ \\
\hline Cartons et papiers & 4,6 & $\%$ \\
\hline Charbon de bois & 9,8 & $\%$ \\
\hline Bouteilles ou verres & 2,9 & $\%$ \\
\hline
\end{tabular}

$\mathrm{MB}$ : matières brutes MS : matières sèches

\section{Tableau 2 : Récapitulation de la caractérisation physico-} chimique des déchets de marché

Les déchets de marché présentent un $\mathrm{pH}$ faiblement acide variant de 5,0 à 6,3. En moyenne, ils ont une conductivité de 2,3 $\mathrm{s} / \mathrm{cm}$ avec une variation de 1,9 à 3,3 $\mathrm{s} / \mathrm{cm}$, ce qui est confirmé par la présence de matières minérales dans nos analyses chimiques (tableau 2).

\begin{tabular}{|l|l|l|l|l|l|l|l|}
\hline \multicolumn{1}{|c|}{ Composition } & $\begin{array}{l}\text { Végétaux } \\
\text { et } \\
\text { animaux }\end{array}$ & Verre & Charbon & $\begin{array}{l}\text { Carton, } \\
\text { papier }\end{array}$ & $\begin{array}{l}\text { Matieres } \\
\text { plastiques }\end{array}$ & Tissus & Métaux \\
\hline $\begin{array}{l}\text { Adam et } \\
\text { Germain } \\
(1997), \text { déchets } \\
\text { ménagers }\end{array}$ & 75 & 2 & 8 & 3 & 7 & 2 & 3 \\
\hline $\begin{array}{l}\text { Résultats de } \\
\text { l'étude sur les } \\
\text { déchets de } \\
\text { marché }\end{array}$ & 79,32 & 2,85 & 9,78 & 4,64 & 3,41 & 0,00 & 0,00 \\
\hline
\end{tabular}

Tableau 3 :Typologie des déchets ménagers et des déchets de marchés en pourcentage

\section{Caractéristiques chimiques des déchets de marché}

Les déchets de marché contiennent en moyenne $78 \%$ de matières organiques. Cette grande proportion vient du fait que les déchets sont constitués principalement de résidus végétaux et animaux (tableau 2), ce qui pourra permettre la production de compost.

Sur l'ensemble des échantillons, on a constaté la présence de 
chlorures $(\mathrm{Cl})$ à un niveau de concentration allant de 1000 à $2500 \mathrm{mg} / \mathrm{kg}$ de matières sèches (moyenne pondérée de 2 286,80 mg/kg). Les ions chlorures existent dans les déchets principalement sous deux formes différentes : $\mathrm{NaCl}$ et autres chlorures retrouvés dans les papiers, cartons, cendres, plastiques, déchets alimentaires et végétaux.

En Haïti, les matières plastiques rencontrées sont les suivantes : polyéthylène haute et basse densité, polystyrène, polychlorure de vinyle, polyéthylène téréphtalate, polypropylène (MDE, 1998). Le polychlorure de vinyle est utilisé dans la fabrication de nombreux objets utilisés par les ménages (Maystre et al., 1999).

Les sulfates $\left(\mathrm{SO}^{2-}\right)$ sont présents à une concentration moyenne de $1520 \mathrm{mg} / \mathrm{kg}$. La principale source est constituée par le gypse utilisé dans les matériaux de construction et par le sulfate de magnésium provenant des minerais. «Les sulfates sont absorbés par les plantes au niveau des racines : le soufre contenu dans les sulfates serait réduit en acides aminés soufrés puis restitué soit par la chaîne alimentaire soit par les déchets grâce aux décomposeurs. 》 (Heinrich et Herat, 1993) «Les sulfates retrouvés dans les déchets analysés proviennent aussi des végétaux (choux) et des animaux, puisque des groupements thiols ont été retrouvés dans les protéines. 》) (Bliefert et Perraud, 2003).

Les nitrates $\left(\mathrm{NO}_{3}{ }^{-}\right)$, dont la concentration ne dépasse pas $500 \mathrm{mg} / \mathrm{kg}$ dans les divers échantillons, ont une concentration moyenne de $284 \mathrm{mg} / \mathrm{kg}$. Ils proviennent essentiellement de l'utilisation d'engrais et de fumiers pour favoriser la croissance des plantes, la culture de légumes et l'élevage intensif sur une petite surface (Bliefert et Perraud, 2003).

Dans les déchets analysés, les légumes peuvent constituer une source de nitrates non négligeable.

La présence de l'ammonium $\left(\mathrm{NH}_{4}^{+}\right)$est faiblement marquée : la moyenne pondérée est de $14,4 \mathrm{mg} / \mathrm{kg}$. L'échantillon SI en contient $72 \mathrm{mg} / \mathrm{kg}$ et dans les autres échantillons, il est présent sous forme de « traces » de 0,8 à $6 \mathrm{mg} / \mathrm{kg}$. Lorsque les plantes dépérissent ou que des animaux meurent, les composés contenant de l'azote sous forme d'urée ou d'amines sont décomposés en ions ammonium (Bliefert et Perraud, 2003). II faut aussi souligner le fait qu'il est courant de voir des gens uriner sur les tas de déchets. Ils apportent ainsi de l'urée qui sera transformée partiellement en ion ammonium.

L'azote est un constituant de base de la biomasse (Heinrich et Herat, 1993) mais avec des teneurs assez faibles. Elément essentiel des protéines, il se retrouve sous des formes variées au sein des déchets analysés.

\section{Les métaux lourds}

Ils sont toujours présents dans les déchets ménagers et des valeurs ont été données par Rousseaux et al. (1990). Leur distribution n'est pas uniforme dans nos échantillons.

Le cuivre est présent seulement dans le premier échantillon et à un niveau très faible. Selon Maystre et al. (1999), une faible quantité de cuivre rencontrée dans les déchets ménagers est due aux cartons d'emballages ( $84 \mathrm{mg} / \mathrm{kg}$ ) et aux résidus carnés et végétaux, respectivement 24 et $28 \mathrm{mg}$ de cuivre par kilo de résidus carnés et végétaux. Le même constat ressort de notre caractérisation.
Les autres métaux lourds sont présents dans tous les échantillons avec des proportions variables.

La teneur en zinc varie de 8 à $152 \mathrm{mg} / \mathrm{kg}$, Or les déchets végétaux, animaux et de papiers ont des teneurs en zinc qui se situent dans cet intervalle (Maystre et al., 1999).

La teneur en plomb varie de 4 à $151 \mathrm{mg} / \mathrm{kg}$. Les sources possibles sont nombreuses : les papiers d'emballages colorés, les peintures, etc. Le plomb est incorporé comme pigments sous forme de chromate $\left(\mathrm{PbCrO}_{4}\right)$, sulfate $\left(\mathrm{PbSO}_{4}\right)$ ou carbonate (céruse $\mathrm{PbCO}_{3}$ ) dans certaines peintures (Tissot, 2004). Les papiers d'emballage ont souvent des teneurs élevées $316 \mathrm{mg} / \mathrm{kg}$ (Maystre et al., 1999), ce qui est tout à fait compatible avec la quantité déterminée lors de notre étude.

La concentration en chrome dans les déchets de marché étudiés varie de 34 à $187 \mathrm{mg} / \mathrm{kg}$. La présence de ce métal dans les ordures ménagères provient essentiellement des verres (59 \% de la teneur en chrome des ordures ménagères). Le chrome est ajouté sous forme d'oxyde chromeux $\left(\mathrm{Cr}_{2} \mathrm{O}_{3}\right)$ ou chromique $\left(\mathrm{CrO}_{3}\right)$ dans la fabrication des verres pour leur donner une couleur verte (Vernus, 2003). En Haïti, ces bouteilles sont utilisées notamment dans la distribution de la boisson appelée « cola ».

\begin{tabular}{|l|l|l|l|l|l|}
\hline \multicolumn{1}{|c|}{ Métaux } & Plomb & Cuivre & Chrome & Zinc & Cobalt \\
\hline $\begin{array}{l}\text { Sources } \\
\text { ordureur moyenne dans les } \\
\text { France (ADEMÈres en } \\
\text { consulté en 2005, } \\
\text { www.recy.net) }\end{array}$ & 800 & 1048 & 183 & 1000 & 113 \\
\hline $\begin{array}{l}\text { Teneur moyenne des } \\
\text { déchets de marché de la } \\
\text { commune de Port-au- } \\
\text { Prince }\end{array}$ & 33,8 & 2,33 & 107,33 & 61,3 & 4 \\
\hline
\end{tabular}

Tableau 4 :Teneur en métaux lourds des ordures ménagères en France et des déchets de marchés de Port-au-Prince en $\mathrm{mg} / \mathrm{kg}$

La concentration en cobalt est très faible, de I à $6 \mathrm{mg} / \mathrm{kg}$ de matière sèche. II proviendrait principalement des matières plastiques (avec $30 \%$ de l'apport total en cobalt) et des papiers cartons (29\%). Le cobalt intervient en effet dans la fabrication des matières plastiques comme pigment bleu sous la forme d'aluminate de cobalt ( $\left.\mathrm{CoO}, \mathrm{Al}_{2} \mathrm{O}_{3}\right)$. Le cobalt des papierscartons peut être raisonnablement attribué aux encres d'imprimerie dans lesquelles il est incorporé comme pigment ou siccatif sous forme de complexes. La concentration en manganèse varie de 38 à 138 mg/kg de matière sèche. Ce métal est utilisé dans la fabrication de piles électriques et des verres sous forme de différents oxydes de manganèse $\mathrm{MnO}$, $\mathrm{Mn}_{2} \mathrm{O}_{3}, \mathrm{MnO}_{2}, \mathrm{Mn}_{3} \mathrm{O}_{4}$ (Vernus, 2003), mais aussi pour l'obtention de nombreux aciers qui en se corrodant libèrent leurs constituants.

Nous donnons, à titre d'information, les teneurs en métaux lourds des ordures ménagères françaises et celles des déchets des deux marchés étudiés dans la République d'Haïti (tableau 4). Seule la teneur en chrome dépasse le seuil tolérable, selon les normes de la Wallonie, pour que les déchets soient compostables (tableau 5). 


\begin{tabular}{|l|l|l|l|l|}
\hline \multicolumn{1}{|c|}{ Métaux } & Plomb & Chrome & Zinc & Cobalt \\
\hline $\begin{array}{l}\text { Nources } \\
\text { (Thones wallones }\end{array}$ & 120 & 90 & 300 & 70 \\
\hline $\begin{array}{l}\text { Teneur moyenne des } \\
\text { déchets de marché de } \\
\text { la commune de Port- } \\
\text { au-Prince }\end{array}$ & 33,8 & 107,33 & 61,3 & 4 \\
\hline
\end{tabular}

Tableau 5 : Teneur en métaux lourds des déchets de marché de Port-au-Prince comparées aux normes de compostage de Wallonie en $\mathrm{mg} / \mathrm{kg}$

D'une manière générale, on peut dire que la présence des métaux lourds dans les déchets est souvent due à des pigments utilisés dans les papiers colorés d'emballages (Maystre et al., 1999). De plus, le pétrole et le charbon contiennent beaucoup d'éléments chimiques, dont des métaux lourds en quantités très variables (Bliefert et Perraud, 2003).

Les papiers d'emballages colorés et les verres rencontrés dans nos observations constituent les principales sources des métaux lourds déterminés (tableau2).

\section{Conclusion}

Les déchets urbains des deux marchés observés contiennent en moyenne plus de $70 \%$ de résidus végétaux et animaux. Ils sont donc facilement biodégradables. Deux facteurs sont favorables pour mettre en place la technique de compostage pour ces déchets : l'humidité, qui dépasse $70 \%$, et les faibles teneurs en métaux lourds, qui sont très inférieures à celles des déchets ménagers utilisés dans d'autres pays pour la fabrication du compost à partir de déchets ménagers. Une étude sur les possibilités techniques, économiques, sociales et commerciales de fabrication de compost à partir de ces déchets de marchés est à entreprendre. Elle permettrait une amélioration de la gestion des déchets à Port-au-Prince et elle constituerait une prise en considération réelle des questions environnementales en Haiti. L'utilisation de ces composts dans l'agriculture, qui est l'activité économique principale de la population, aurait des effets très bénéfiques pour la conservation des sols. La bio-méthanisation de la fraction organique, quoique très intéressante dans la lutte contre l'effet de serre, reste encore une technologie difficilement adaptable dans un pays comme Haïti, du fait des forts investissements qui sont nécessaires pour mettre en place des installations performantes.

\section{Références bibliographiques}

Adam, P. et Germain, F. (1997) : Manuel technique de gestion des déchets. Port-au-Prince : Cooperative Housing Foundation (CHF). $59 \mathrm{p}$.

ADEME (2002) : Etat des lieux et perspectives de recyclage des déchets issus des plumes et duvets en France [en ligne]. 54 p. disponible sur www.recy.net, consulté en mars 2005.

Bliefert C. et Perraud R., (2003) : Chimie de l'environnement. Editions de Boeck. Paris., 477 p.
Charnay F, (2005) : Compostage des déchets urbains dans les pays en développement : élaboration d'une démarche méthodologique pour une production pérenne de compost. Thèse. Université de Limoges. $277 p$.

Thonart P., Lardinois M., Kapepula D.,Hiligsmann S., (2002) : La problématique de la gestion des dechets ménagers en République d'Haïti. Centre wallon de biologie industrielle. Faculté des sciences agronomique de Gembloux, Université de Liège. 35 p.

Heinrich D. et Hergt M., (1993) :Atlas de l'écologie. Le livre de poche - Collection La Pochothèque. 288 p.

Maystre L.Y. et al. (1994): Déchets Urbains Nature et Caractérisation, Presse polytechnique et universitaires Romandes. Lausanne. 219 p. Ministère de l'Environnement d'Haïti (MDE) (1998) : Etude sur les déchets plastiques et les possibilités de valorisation. p 88 .

Mustin M. (1987) : Le compost français. François DUBUSC. Paris. 953 p. Rousseaux P., Navarro A. et Vermande P. (1990) : Distribution des sept principaux métaux lourds dans les constituants des ordures ménagères. CE BEDEAU, La Tribune de l'eau, $n^{\circ}$ 5, Vol. 42, nº 54I, p. 17 à 25.

Tissot B. (2004) : Sécurité sanitaire et gestion des déchets : quels liens ? Réflexions et propositions. Rapport à la Faculté de sciences. Paris. $188 p$

Vernus K. (2003) : Déchets et santé. École nationale vétérinaire de Lyon. $17 \mathrm{p}$.

Zahrani F., Naquin P., Ngnikam E. (2006) : Pré-collecte des déchets ménagers dans les pays en développement : comment évaluer les actions menées?. Déchets, Sciences \& Techniques, n 43, pp. 31-39. 